\title{
The role of vision in the science and art of the putting stroke in the sport of golf
}

\begin{abstract}
Vision and visual processing are essential when executing a skill in sports that involves striking a ball and having it reach its intended target with the correct speed and trajectory. Successful putting in the sport of golf requires visual tasks such as aiming, determining the slope of the green, choosing the correct area of focus, wearing the best possible visual correction, and other psychological factors that may affect visual processing. This article reviews, integrates, and clarifies the fact and fiction of visual research, the science of vision and visual processing, and provides a summary of methods that may be beneficial for coaching, practicing, enjoying, and competing in golf of which putting on the green is a major component.
\end{abstract}

Keywords: golf, putting, putting science, sports vision, sports visualization, sensorimotor, sports visual processing, target aiming, depth perception in sports, slope determination, plumb bobbing, target gaze position, gaze pattern, gaze variability, target focusing, perception-action coupling, sports visual devices, sports contrast sensitivity
Volume 8 Issue 3 - 2018

\author{
Brian M DeBroff \\ Department of Ophthalmology and Visual Science, Yale \\ University School of Medicine, USA
}

Correspondence: Brian M DeBroff, Department of Ophthalmology and Visual Science, Yale University School of Medicine, 46 Prince Street, Suite 404, New Haven, CT 06519, USA, Tel +203-375-5819; +203-913-1605, Email bmdmd@aol.com

Received: May 02, 2018 Published: May 17, 2018

\section{Introduction}

Golf is a popular sport that requires coordination, eye-hand coordination, and complex sensory motor skills to judge distance, speed, and conditions such as slope, grass height, and subtle angles and curvatures that can impact ball flight, trajectory, and roll. Experience, acquired skill, and psychological aspects can impact performance. According to the PGA, approximately $40 \%$ of all strokes in a given golf round involve putting the ball on the golf green. The art and skill of putting requires complex sensorimotor input for successful execution. This article reviews the contribution of scientific research in vision and its impact on successful putting in the sport of golf. Putting performance is dependent upon determining the proper putter speed required to displace the ball from its resting position to the hole taking into consideration the friction between the ball and grass, the vertical displacement of the hole relative to the ball, the distance the ball must travel to reach the hole, and the direction and magnitude of the break of the green. Vision and proper visual processing by the brain are vital components to successful execution of this complex skill. Electroencephalography studies have demonstrated that expert golfers demonstrate greater stimulation of regions of their brains as compared with novice counterparts, indicating greater sensory information processing. ${ }^{1}$

In addition, the golfer must also execute a putting stroke where, at impact, the putter-head has horizontal velocity in the direction of the target line and the plane of the putter-face is perpendicular to that line. ${ }^{2}$ Visualizing and executing a perpendicular putter-face angle is imperative. One study compared putter face angle, putter path, and putter impact point and found that the putter face angle was most important of the three. ${ }^{3}$ Dr. Craig Farnsworth, a vision training specialist also known at The Putt Doctor, who has coached numerous pro tour golfers, contends that many players will compensate for a putt they believe to have been pulled by opening the blade at impact. ${ }^{4}$ Harold Swash aka the European Putt Doctor, contends that for consistent putting it is imperative for the blade of the putter to be square to the target at both golf ball address and golf ball strike position. ${ }^{4}$ Mallet style putters have become increasingly popular due to the tendency for the face to remain square throughout the swing. Still, practice and visualization of a square putter head face are very important aspects of the putting stroke. Experienced golfers have been shown to have more predictable results by maintaining smooth and similar putting motions. ${ }^{5}$

\section{Aiming the putt}

An important aspect of putting success is determining the best line or path the ball will take on the way to the hole. Proper aim is essential to successful putting. Binocular vision has been found to be advantageous when performing a complex sensorimotor task, such as golf putting. ${ }^{6}$ Binocular vision improves stereopsis which is the ability of the visual system to use horizontal retinal-image disparity information to contribute to the overall perception of relative depth and three-dimensional shape. ${ }^{7}$ Binocular vision is not, however, useful when sighting your target. For example when a golfer stands behind the ball and determines the line of the putt, only the dominant eye should be utilized. Accurate sighting, just as sighting when aiming a gun, is most accurate when utilizing the dominant eye. There is a Tiger Wood's anecdote in which his father told him to look at the hole, say "click" to take a mental photograph, and then strike the putt with the image of the photograph in mind. ${ }^{8}$ Lexi Thompson, a top contender on the LPGA tour, started putting with her eyes closed as a practice drill and it worked so well that she has been using it successfully in official tournaments. Sometimes the mental image works better than reality. In fact, when golfers do not utilize sightings from behind the ball, but rather direct their aim while over the ball, there tend to be errors in alignment that lead to deviation of the actual target line. In one study, over $88 \%$ of right handed participants demonstrated eye nonparallel alignment during putting, mostly to the right of the target. ${ }^{4}$ Another study found that common lateralized attentional biases lead to a rightward bias for putting error in novice golfers. ${ }^{9}$ Other aspects of aiming have been studied. Robert Grober, a former physics 
professor at Yale University who says he "studies golf as a hobby," has expanded on the well-known axiom that putters should aim for a point just beyond the hole. ${ }^{10} \mathrm{He}$ also recommends that golfers take a step to the left and another to the right, and look at the putts from those points. The lines will meet, forming a diamond beyond the hole which should serve as the intended target. ${ }^{10}$

\section{Determining slope/plumb bobbing}

Determining the best line that the golf ball will travel to the hole includes being able to determine the slope of the green. In "The Putting Bible" Dave Pelz describes how amateurs typically play only one-third of the true break and professionals typically play only twothirds of the true break. ${ }^{11}$ There is no accepted method for determining the slope of the green. One method that has been proposed and utilized by some golfers, including PGA professionals, is the plumbbob method. Pelz in 2000, wrote that one in seven golfers uses the plumb-bob method for reading theslope. ${ }^{12}$ In 1992, Foston described plumb bobbing as a technique in which the golfer stands behind the golf ball and straddles an imaginary line that bisects the stance of the golfer, the golf ball and finally the hole itself. By suspending the putter at arm's length and allowing gravity to pull the shaft in vertical alignment, using the dominant eye, the golfer aligns the bottom of the putter shaft with the center of the golf ball. Supposedly, if there is slope, the shaft will be observed to be on the high side of the hole. ${ }^{13}$ Pelz contends that the only condition in which the plumb bob method will correctly indicate slope is when a green is consistently graded from the golfer to past the hole. This certainly is not the case for most greens a golfer will encounter. A laboratory study evaluating the plumb bob method found the results "highly inconsistent." 2 The slope beneath the golfer's feet had the largest influence on the perceived slope. All golfers should realize, however, that the slope beneath the golfers feet does not predict the slope between the ball and the hole. ${ }^{2}$ The effect of plumb bobbing on creating relaxation or confidence may have a limited positive effect.

\section{Effect of putting grip on eye and head movements}

A study by Hung at the Department of Biomedical Engineering at Rutgers compared the effect of 3 different putting grips (conventional, cross-handed, and one-handed) on variations in eye and head movements during the putting stroke. Head and eye movements were the greatest with the conventional grip and percentage of putts made by novice players was highest with the cross-handed grip. ${ }^{14}$

\section{Psychological factors that may affect visual processing}

Cognitive anxiety or the pressure to achieve good results can result in a negative relationship with performance. Self confidence has been shown to have a positive relationship with performance. A putter visualizing negative outcome images was found to be detrimental to putting performance. ${ }^{15}$

\section{Area of focus when putting}

Jessica Witt, a cognitive psychologist, found that putters can improve their accuracy by up to 10 percent by using a trick to make a hole seem larger on the green. A common method involves surrounding the hole with smaller circles, which makes the hole appear bigger than it actually is. ${ }^{16}$ One question that has been in the spotlight recently is what the best gaze position during the putting process? Studies have determined that with the acquisition of putting skill, gaze control is characterized by an economy in the number of gaze shifts between the hole and the ball. ${ }^{17}$ Professional golfers use a more economical gaze pattern consisting of fewer fixation changes. ${ }^{18}$ Smith, a sports psycho-physiologist, recommends that immediately before the execution of the putt, golfers should narrow their focus to the putter and the ball. Reducing the variability of your gaze increases the likelihood of successful putts. He recommends that a golfer should concentrate on the ball and continue to look at the same spot after striking the ball. ${ }^{19}$ Typically golf instructors and coaches teach players to "keep their eye on the ball." In fact, Pelz in Putt Like the Pros, recommends a seven step routine which includes a series of looking at the target to the target line and finally in step seven looking back at the ball. ${ }^{20}$ Recent studies, however, have demonstrated that successful putting results from a greater focus on the hole (target) than the ball. ${ }^{21}$

Some professional golfers, such as Jordan Spieth, Loius Oosthuizen, and Colin Montgomerie have utilized a technique of looking at the hole when putting, especially for putts under 10 feet. Potential benefits of this technique include an improved perception-action coupling, allowing the brain to use environmental clues of distance and break while providing a complementary action of changing the focus of attention and thus avoiding the yips (involuntary wrist spasms that make one unable to execute a smooth stroke). ${ }^{8}$ Mackenzie in 2011 found comparable results in variability of the putter face angle, the stroke path, and the impact spot for both looking at the ball and looking at the hole. The only difference was less variability in putter head speed. ${ }^{22}$ Alpenfels et al. ${ }^{23}$ performed a test using 40 golfers with a range of handicaps. When looking at the hole instead of the ball, golfers of all handicaps putted more effectively, and were less likely to move their heads or decelerate the putter head before impact. Additional studies with beginning golfers found no significant difference in multiple putts over different types of terrain whether they looked at the hole or the ball. ${ }^{24-26}$

\section{Visual aids and devices}

Visual acuity plays a very important role in the successful execution of putting. Previous studies have demonstrated that retinal defocus from either presbyopia or inappropriate refractive correction can lead to poorer putting results. ${ }^{27,28}$ Maximizing the best visual acuity with corrective lenses or contacts is important for aiming, reading the speed of the greens, and determining the slope. Many golfers remove sunglasses when putting, especially polarized sunglasses which limit glare and thus hinder the detection of subtle undulations or break of the green. Most sunglasses decrease contrast sensitivity. Many golfers have complained that sunglasses hinder depth perception and limit tracking of the golf ball. To minimize the effect of polarization on contrast sensitivity, some manufacturers are combining the technology of high definition lenses with polarization. In such a manner, these sunglasses offer protection from the sun without impacting visual acuity on the greens. Some manufacturers have developed technology specifically designed for golf including the Oakley Flak PRIZM sunglasses which reportedly highlight or mute certain colors and differ for different sports depending on the color of the most common background of the sport (e.g. sky, grass, or snow). Also a system of True Digital Edge developed by Oakley was developed to modify the curvature of the glasses' lens to help reduce peripheral distortions. Rudy Project Rydon Golf sunglasses were developed with ergonomic design, air ventilation control, and photo chromic lenses specifically designed for golf. Under Armour Igniter Storm Sunglasses were designed to withstand high speed motion activities (e.g. golf swing) and less distortion of peripheral vision. 
Adidas has Tour Pro Sunglasses that have a silver mirror lens with light stabilizing technology to make golf play clearer and crisper. Some of the features that golfers seek in sunglasses are polycarbonate lenses, $100 \%$ UV protection, polarization or a combination or polarized/non-polarized, snug fit to prevent sliding or require constant adjustment, and less prone to fogging in conditions of humidity or perspiration. In addition, comfort is of paramount importance. In terms of shading, brown and amber tints are often best for viewing a white ball against a blue sky.

Athletes in other sports have avoided sunglasses because of reduced contrast sensitivity (e.g. tracking a ball). One visual aid that has been utilized in sports like football, lacrosse, baseball, and even tennis is Eye Black grease which has been shown to reduce glare in conditions of sunlight and improve contrast sensivity, ${ }^{29-31}$ but has not been evaluated or popularized for golf. The improved contrast sensitivity is felt to be helpful in seeing balls flying toward the athlete. It has been suggested that perhaps golf spectators rather than players may benefit from wear Eye Black grease. Some golfers also perform more poorly with bifocal or progressive lenses when putting, as they may be looking through the bottom (close vision) part of the glasses resulting in defocus. Special bifocal glasses can be made that work better for golf: setting the bifocal portion low enough in the glass frame so that it does not interfere with putting or placing a bifocal in one lens only in the bottom peripheral quadrant. In such a manner one can tilt or lift the glasses when they are need for example keeping score. ${ }^{32}$ For those golfers with very little accommodative amplitude, bifocals set a distance of five feet could be considered to assist in properly addressing the golf ball. ${ }^{32}$

\section{Conclusion}

Being able to precisely visualize the most accurate ball pathway after ball displacement is a key factor to success in putting. Vision plays an important role in assisting a golfer to maintain a square putter head stroke, ascertain the best line or path of the ball, accurately determine the slope of the green, sustain positive visual images, and optimize gaze control during the putting process. In addition, maximizing the best optical correction for different playing conditions is important. Future technologies and visual aids will hopefully continue to reduce contrast sensitivity and glare and improve visualization of subtle contour changes of the putting green under different playing conditions. In addition to these visual factors, fine eye hand coordination and precise physical execution are needed for putting success.

But even with the most consistent stroke and constant conditions of the green, normal variability will effect whether a putt leads to the ball finding the bottom of the cup. Biomechanical studies that use mechanized models (e.g. a robotic type putting apparatus) that precisely control for green and ball speed, ball acceleration, momentum, and velocity, green slope and elevation, and friction, found as much as a $15 \%$ error in biomechanically equivalent putts. ${ }^{33}$ A $15 \%$ directional error is magnified significantly with putts of increasing lengths. Finally, components of chance and luck, also known at the "rub of the green," characterize the degree of unpredictability in even the best of putts by the best of putters. By controlling as many of the known factors that have been shown to be successful, however, players will potentially improve their success in lowering the number of putts per round, golf scores, and enjoyment of the sport. This paper describes results of multiple scientific studies regarding vision and summarizes the best known discoveries in the science of sight as related to increasing putting proficiency and maximizing putting success.

\section{Acknowledgments}

None.

\section{Conflict of interest}

The author declares that there is no conflict of interest.

\section{References}

1. Baumeister J, Reinecke $\mathrm{K}$, Liesen $\mathrm{H}$, et al. Cortical activity of skilled performance in a complex sports related motor task. Eur J Appl Physiol. 2008;104(4):625-631.

2. Mackenzie SJ, Sprigings EJ. Evaluation of the plumb-bob method for reading greens in putting. J Sports Sci. 2005;23(1):81-87.

3. Karlsen J, Smith G, Nilsson J. The stroke has only a minor influence on direction consistency in golf putting among elite players. $J$ Sports Sci. 2008;26(3):243-250.

4. MacKay J. An analysis of eye and club face alignment at address in putting. In: Science and Golf $V$. Proceedings of the World Scientific Congress of Golf. Crews D, Lutz R., editors. 1998; Chapter 27: p. 202-207.

5. Choi JS, Kim HS, Yi JH, et al. Kinematic Analysis of Golf Putting for Elite and Novice Golfers. The Impact of Technology on Sport. CRC Press, 2008. p. 277-282.

6. Bulson R, Ciuffreda KJ, Ludlam DP. Effect of binocular vs. monocular viewing on golf putting accuracy. J Behav Optom. 2009;20(2);31-34.

7. Howard IP, Rogers BJ. Binocular Vision and Stereopsis. New York, NY: Oxford University Press; 1995.

8. Young A. The Practice Manual, The Ultimate Guide for Golfers. Charleston, SC: CreateSpace Publishing; 2015.

9. Robert R, Turnbull O. Putts that get missed on the right: Investigating lateralized attentional biases and the nature of putting errors in golf. $J$ Sports Sci. 2010;28(4):369-374.

10. Grober RD. "The Geometry of Putting on a Planar Surface". ARXIV, Yale University 2011.

11. Pelz D, Frank JA. Dave Pelz's Short Game Bible: Master the finesse swing and lower your score. New York, NY: Broadway; 1999.

12. Pelz, D. Dave Pelz's Putting Bible. New York, NY: Doubleday; 2000.

13. Foston, P. The Encyclopedia of Golf Techniques: The Complete StepBy-Step Guide to Mastering the Game of Golf. Philadelphia, PA: Courage Books; 1992.

14. Hung G. Effect of putting grip on eye and head movements during the golf putting stroke. The Scientific World Journal. 2003;3:122-137.

15. Taylor JA, Shaw DF. The effects of outcome imagery on golf putting performance. J Sports Sci. 2002;20(8):607-613.

16. Witt JK, Linkenauger SA, Bakdash JZ, et al. Putting to a bigger hole: Golf performance relates to perceived size. Pychonomic Bull \& Rev. 2008;15(3):581-585.

17. Vickers JN. Gaze control in putting. Perception. 1992;21(1):117-132.

18. Campbell MJ, Moran AP. There is more to green reading than meets the eye! Exploring the gaze behaviors of expert golfers on a virtual putting task. Cognitive Processing. 2014;15(3):363-372.

19. Smith MF. The role of physiology in the development of golf performance. Sports Med. 2010;40(8);635-655. 
20. Pelz D, Mastroni N. Putt Like the Pros. New York, NY: Harper Perennial; 1989.

21. Fairchild MD, Johnson GM, Babcock J, et al. Is your eye on the ball? Eye tracking golfers while putting. Science in Golf Competition: Rochester, NY: Rochester Institute of Technology, Unpublished manuscript: 2001.

22. Mackenzie SJ, Foley SM, Adamczyk AP. Visually focusing on the far versus the near target during the putting stroke. J Sports Sci. 2011;29(12):1243-1251.

23. Alpenfels E, Christina B, Heath C. Instinct Putting, Putt Your Best Using the Breakthrough, Science-Based Taget Vision Putting Technique. New York, NY: Gotham Books; 2008.

24. Bowen RT. Putting errors of beginning golfers using different points of aim. Research Quarterly, Am Soc Health Phys Eds 2013:39(1).

25. Aksamit G, Husak W. Feedback influences on the skill of putting Sage Journals. 1983;56(1):19-22.

26. Gott E, McGown C. Effects of a combination of stances and points of aim on putting accuracy. Sage Journals. 1988;66(1).
27. Hung GK, Ciuffreda KJ, Selenow A, et al. Effect of wearing progressive lenses on eye and head movements during the golf putting stroke. J Behav Optom. 2006;17:115-119.

28. Bulson RC, Ciuffreda KJ, Hung GK. The effect of retinal defocus on golf putting. Ophthal Physiol Opt. 2008;28(4):334-344.

29. DeBroff BM, Pahk PJ. The ability of periorbitally applied antiglare products to improve contast sensitivity in conditions of sunlight exposure. Arch Ophthalmol. 2003;121(7):997-1001.

30. DeBroff BM. The Ophthalmic science behind eyeblack grease and its use in professional and youth athletics. Advances in Opthalmology \& Visual Science. 2016;4(2): 00100.

31. DeBroff BM. The Eye Black Study. Dexter, MI: Dunlap Goddard; 2013.

32. Lampert LD. The Pro's Edge. Vision Training for Golf. Boca Raton, FL: Saturn Press: 1998.

33. Santiago-Martinez P. A mechanical based approach for putt distance optimization. HIM. 2015;1741. 